\title{
Variational method for lattice spectroscopy with ghosts
}

\author{
Tommy Burch ${ }^{a}$, Christof Gattringer ${ }^{b}$, Leonid Ya. Glozman $^{b}$, Christian Hagen $^{a}$, and C. B. Lang ${ }^{b}$ \\ (Bern-Graz-Regensburg (BGR) collaboration) \\ ${ }^{a}$ Institut für Theoretische Physik, Universität Regensburg \\ D-93040 Regensburg, Germany and \\ ${ }^{b}$ Institut für Physik, FB Theoretische Physik Universität Graz \\ A-8010 Graz, Austria
}

(Dated: November 23, 2005)

\begin{abstract}
We discuss the variational method used in lattice spectroscopy calculations. In particular we address the role of ghost contributions which appear in quenched or partially quenched simulations and have a non-standard euclidean time dependence. We show that the ghosts can be separated from the physical states. Our result is illustrated with numerical data for the scalar meson.
\end{abstract}

PACS numbers: 11.15.Ha, 12.38.Gc

\section{QUENCHED CORRELATORS AND THEIR SPECTRAL REPRESENTATION}

Spectroscopy of excited states is still a quite challenging problem for lattice QCD. The reason is that euclidean two-point functions have a sum of infinitely many exponentials in their spectral representation and it is a non-trivial task to extract the subleading terms corresponding to the excited states. For quenched or partially quenched calculations the situation is complicated further by additional unphysical contributions: When ignoring the fermion determinant, the $\eta^{\prime}$ also becomes a Goldstone boson, and this massless flavor singlet state can couple in various processes through hairpin diagrams. The corresponding effects were studied in [1] for the quenched scalar propagator. It was demonstrated that the $\eta^{\prime}$ leads to additional contributions to the scalar propagator which have a negative sign and a non-standard $t$-dependence. Such terms, often referred to as "ghosts", compete with the exponential decay coming from the scalar, and make the extraction of the scalar mass cumbersome. For the nucleon system the effects of ghost states and the problems they cause in the extraction of the Roper resonance were discussed in [2].

In principle, in a quenched calculation, correlators for all quantum numbers may be infested by ghosts, possibly coming from bound or scattering states with $\eta^{\prime}$, or states involving even several $\eta^{\prime}$. Thus, one has to find tools for reliably removing these contributions, in particular, if one is interested in excited states, where the influence of the ghost states is even more devastating.

A powerful tool, developed for the analysis of excited states, is the variational method [3, 4]. The main idea is to work with several linearly independent interpolators $O_{i}, i=1,2, \ldots r$, all with the quantum numbers of the desired state, and to compute the cross-correlations

$$
C(t)_{i j}=\left\langle O_{i}(t) \bar{O}_{j}(0)\right\rangle, \quad i, j=1,2, \ldots r .
$$

In full euclidean lattice QCD the correlators have the spectral decomposition

$$
C(t)_{i j}=\sum_{n=1}^{\infty} v_{i}^{(n)} v_{j}^{(n)^{*}} e^{-t V_{n}},
$$

where the coefficients $v_{i}^{(n)}$ are given by

$$
v_{i}^{(n)}=\left\langle 0\left|O_{i}\right| n\right\rangle .
$$

In Eq. (2) $V_{n}$ denotes the energy of the state $|n\rangle$.

In the presence of ghosts we must augment the decomposition (2) by additional terms which take into account the possible negative sign and the different $t$-dependence of the ghost contributions. Since, in principle, there are infinitely many ghost states, their contribution also gives rise to an infinite tower of states

$$
\sum_{n=1}^{\infty} w_{i}^{(n)} w_{j}^{(n)^{*}} f^{(n)}(t) e^{-t W_{n}} .
$$

Here $W_{n}$ is the energy of the $n$-th ghost state and the function $f^{(n)}(t)$ describes the non-exponential part of its euclidean time dependence, taking into account also a possible negative sign. We stress that for our analysis we do not have to know the precise form of $f^{(n)}(t)$. Certainly it is not simple to present a form which encompasses all possibilities that might occur. However, to give an example, we remark that the linear behavior $f(t)=-(a+b t)$ was suggested for the system considered in 2].

In order to combine the contribution (44) from the ghosts and the original decomposition (2), we represent our correlation matrix as

$$
C(t)_{i j}=\sum_{n=1}^{\infty} a_{i}^{(n)} a_{j}^{(n)^{*}} H^{(n)}(t) e^{-t E_{n}},
$$

where

$$
\begin{aligned}
& H^{(n)}(t)=1, E_{n}=V_{n_{P}} \text { for proper states } \\
& H^{(n)}(t)=f^{\left(n_{G}\right)}(t), E_{n}=W_{n_{G}} \text { for ghosts. }
\end{aligned}
$$


Here we have ordered the energy levels such that (assuming non-degenerate values for the $E^{(n)}$ )

$$
0<E_{1}<E_{2}<E_{3} \ldots,
$$

implying that the ghost state energies are interleaved with the energies of proper states. A typical situation would be

$$
0<V_{1}<W_{1}<V_{2} \ldots,
$$

where the energy $W_{1} \equiv E_{2}$ of the first ghost state comes after the ground state energy $V_{1} \equiv E_{1}$, but is below the first excited energy level $V_{2} \equiv E_{3}$. In such a case the ghost contribution overlays the exponential decay from $V_{2}$ and makes the extraction of this number from a single correlator quite difficult.

\section{VARIATIONAL METHOD WITH GHOST CONTRIBUTIONS}

Having generalized the formula for the spectral representation from the original form (2) to the ansatz (5), which is capable of describing also the ghost contributions, we can now proceed as in [4]. In particular, we consider the generalized eigenvalue problem

$$
C(t) \vec{\psi}^{(k)}=\lambda(t)^{(k)} C\left(t_{0}\right) \vec{\psi}^{(k)} .
$$

On the right-hand side we use the correlation matrix at some fixed $t_{0}<t$ to normalize the eigenvalue problem. We now show that the eigenvalues $\lambda(t)$ of the generalized eigenvalue problem are given by

$$
\begin{aligned}
& \lambda^{(k)}(t)=e^{-\left(t-t_{0}\right) E_{k}} \frac{H^{(k)}(t)}{H^{(k)}\left(t_{0}\right)}\left[1+\mathcal{O}\left(e^{-\left(t-t_{0}\right) \Delta_{k}}\right)\right] \\
& =\left\{\begin{array}{l}
e^{-\left(t-t_{0}\right) V_{k}}\left[1+\mathcal{O}\left(e^{-\left(t-t_{0}\right) \Delta_{k}}\right)\right] \text { for proper states, } \\
e^{-\left(t-t_{0}\right) W_{k}} \frac{f^{(k)}(t)}{f^{(k)}\left(t_{0}\right)}\left[1+\mathcal{O}\left(e^{-\left(t-t_{0}\right) \Delta_{k}}\right)\right] \text { for ghosts, }
\end{array}\right.
\end{aligned}
$$

where $E_{k}$ is the energy of the $k$-th state (proper state or ghost) and $\Delta_{k}=E_{r+1}-E_{k}$ is the difference to the mass of the $r+1$-st state, $r$ being the number of interpolators used for the correlators.

The proof proceeds by considering the generalized eigenvalue problem

$$
\widetilde{C}(t) \vec{\psi}^{(k)}=\lambda(t)^{(k)} \widetilde{C}\left(t_{0}\right) \vec{\psi}^{(k)},
$$

for the hermitian $r \times r$ matrix $\widetilde{C}(t)$, obtained by truncating the spectral sum after the $r$-th term,

$$
\widetilde{C}(t)_{i j}=\sum_{n=1}^{r} a_{i}^{(n)} a_{j}^{(n)^{*}} H^{(n)}(t) e^{-t E_{n}}, \quad i, j=1,2, \ldots r .
$$

We assume that the coefficients $a_{i}^{(n)}, i, n=1,2, \ldots r$, form a matrix of full rank. For the proper states the $a_{i}^{(n)}$ are given by (3). For linearly independent interpolators $O_{i}$, that couple to the first $r$ states, full rank follows from the fact that the states $|n\rangle$ are orthonormal. For the quenched approximation one expects that the ghost states are linearly independent of the proper states such that again for linearly independent $O_{i}$ the matrix $a_{i}^{(n)}$ has full rank.

Inserting (12) in (11) one finds that for all $i$

$\sum_{n=1}^{r} a_{i}^{(n)} \rho^{(n, k)}\left[H^{(n)}(t) e^{-t E_{n}}-\lambda(t)^{(k)} H^{(n)}\left(t_{0}\right) e^{-t_{0} E_{n}}\right]=0$, with the coefficients $\rho^{(n, k)}$ given by

$$
\rho^{(n, k)}=\sum_{j=1}^{r} a_{j}^{(n)^{*}} \psi_{j}^{(k)} .
$$

From the full rank of $a_{i}^{(n)}$ it follows that the vectors $a_{i}^{(n)}, n=1,2, \ldots r$, are linearly independent, implying

$$
\rho^{(n, k)}\left[H^{(n)}(t) e^{-t E_{n}}-\lambda(t)^{(k)} H^{(n)}\left(t_{0}\right) e^{-t_{0} E_{n}}\right]=0 \quad \forall n .
$$

Using the linear independence of the eigenvectors $\vec{\psi}^{(k)}$ and the full rank of $a_{j}^{(n)}$, it follows that for each $n$ there exists a $k$, such that $\rho^{(n, k)}$ is non-vanishing. Consequently, Eq. (15) implies that the eigenvalues of the reduced problem (11) are given exactly by the leading term in (10). The full matrix $C(t)$ is obtained from $\widetilde{C}(t)$ by adding terms of $\mathcal{O}\left(\exp \left(-t E_{r+1}\right)\right)$ which can be treated perturbatively [4], giving rise to the correction in (10).

Before we come to discussing an application of the generalized variational method, let us briefly address once more the implications of the result (10): In the leading term each eigenvalue couples to only one state (ghost or proper state). Thus, ghosts can be cleanly disentangled from the proper states, up to the correction term $\mathcal{O}\left(\exp \left(-t \Delta_{k}\right)\right)$. We stress that the form of the euclidean time dependence for the ghosts, $f^{(k)}(t)$, needs not be known or modelled for the application of the method. In the example we discuss below, the ghost state is easily identified by its non-standard $t$-dependence.

We remark that additional information for the identification of the ghost states comes from the standard eigenvalue problem, i.e., when the normalization with $C\left(t_{0}\right)$ is omitted on the right-hand side of (9). Following the arguments given in the appendix of [4], one finds that for the standard eigenvalue problem the eigenvalues are given by

$$
\lambda^{(k)}(t) \propto e^{-t E_{k}} H^{(k)}(t)\left[1+\mathcal{O}\left(e^{-t \delta_{k}}\right)\right] .
$$

Since here the factor $H^{(k)}(t)$ is not normalized by $H^{(k)}\left(t_{0}\right)$, the sign information is not lost and an overall sign indicates a ghost. However, this information is obtained at the cost of a much larger correction term, since in (16) $\delta_{k}$ is the distance to the nearest energy level which is usually much smaller than $\Delta_{k}$ appearing in (10). 

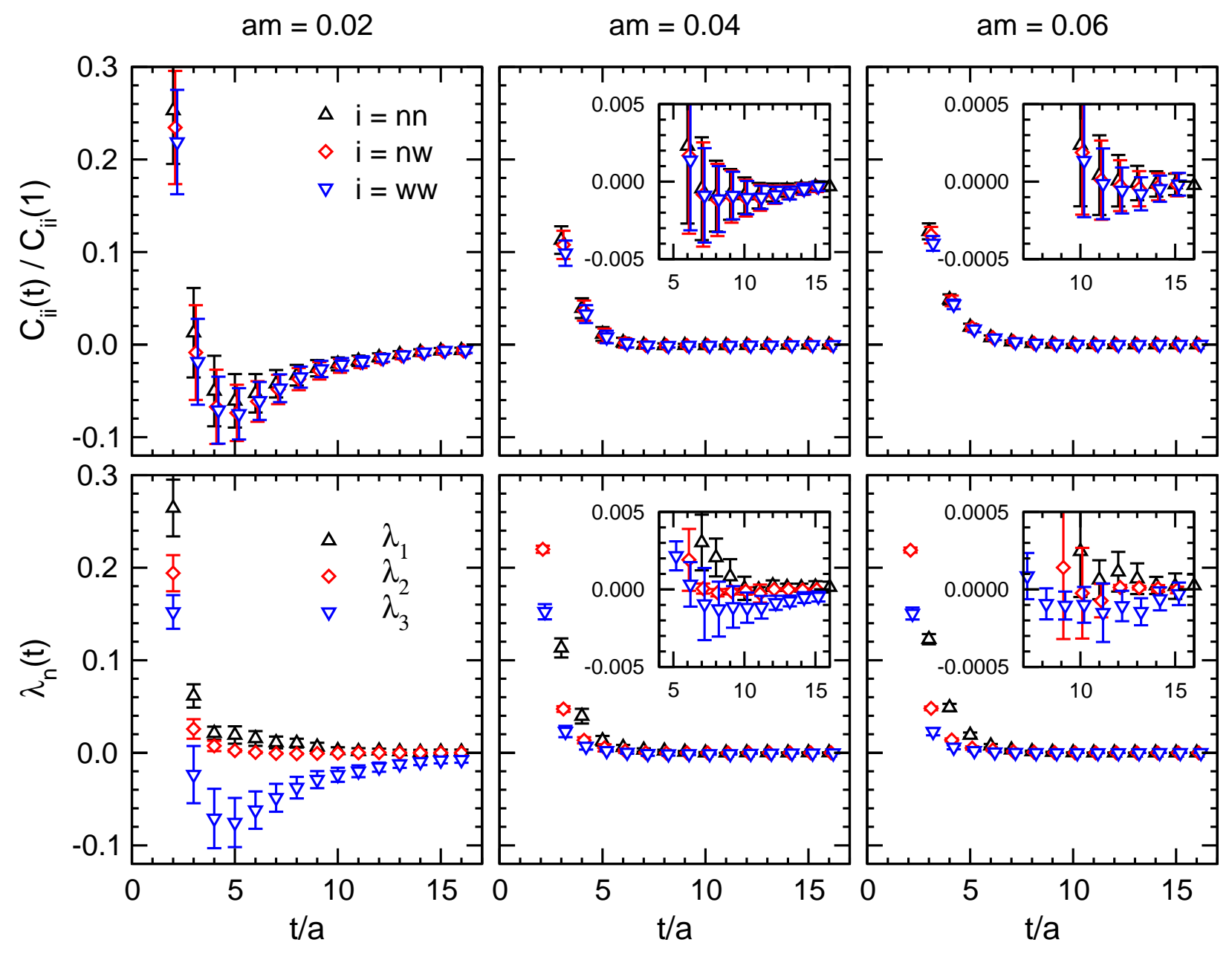

FIG. 1: Euclidean time dependence for the diagonal entries of the correlation matrix (top row) and for the eigenvalues of the generalized eigenvalue problem (bottom row). The quark masses are $a m=0.02,0.04$ and 0.06 (left to right).

\section{ILLUSTRATION OF THE RESULT FOR THE SCALAR MESON PROPAGATOR}

We illustrate our result using the scalar meson as an example. In particular, we work with the three interpolators

$$
\bar{u}_{n} d_{n}, \bar{u}_{n} d_{w}, \bar{u}_{w} d_{w} .
$$

The subscript $n$ denotes a narrow quark source (or sink), while $w$ is used for wide. The narrow and wide quark sources (sinks) are constructed via different amount of Jacobi smearing [5]. Such interpolators with mixed width for the quark sources have been utilized in lattice spectroscopy of excited mesons and baryons and details of the source preparation can be found in [6].

We remark, that the interpolators used here are not meant to resolve the issues currently discussed for the scalar meson (see e.g. 1, 7]). They only serve to illustrate our result for the system where the importance of ghost contributions was first discussed [1]. A detailed analysis of the spectroscopy results for the interpolators (17) will be presented elsewhere.

Using the three interpolators (17) we work with a $3 \times 3$ correlation matrix $C(t)$. We evaluate it on 100 quenched gauge configurations on a $20^{3} \times 32$ lattice using the chirally improved lattice Dirac operator [8]. The gauge action is the Lüscher-Weisz action at $\beta=8.15$, corresponding to a lattice spacing of $a=0.119 \mathrm{fm}$ determined from the Sommer parameter [10]. We present results at three different quark masses $a m=0.02,0.04,0.06$, giving rise to pion masses of $m_{\pi} \sim 400,550$ and $670 \mathrm{MeV}$ [1]. All errors given are statistical errors determined with single elimination jackknife.

In the top row of plots in Fig. 11we show the euclidean time dependence for the diagonal entries of the correlation matrix. We remark that the off-diagonal elements show qualitatively the same behavior, but we omit them in the plot to avoid overcrowding it. For the smallest quark mass the entries of the correlation matrix become negative at $t / a=3$ or 4 , showing the strong influence of the ghost contribution. For $a m=0.04$ the effect is 
still visible, but the correlators become negative only at values $t / a=6$ or 7 . At the largest quark mass the correlators do not become significantly negative, i.e., within error bars are compatible with zero for $t / a>10$. This indicates that the role of the ghost state is much weaker at larger quark masses. This is as expected from the fact, that the mass of the would-be Goldstone particles, and thus of the ghost states, increases very fast with the quark mass.

The bottom row plots show the time dependence for the three eigenvalues of the generalized eigenvalue problem. They behave as expected from formula (10). Only one of them, $\lambda^{(3)}$, shows the behavior characteristic of ghosts. The other two eigenvalues $\lambda^{(1)}$ and $\lambda^{(2)}$ remain positive and are compatible with a single exponential decay. The sign change of $\lambda^{(3)}$ is most prominent at $a m=0.02$ and the overall time dependence is similar to the behavior of the corresponding diagonal elements of the correlation matrix in the top left plot. For $a m=0.04$ the ghost-like behavior of $\lambda^{(3)}$ is still visible, while for $a m=0.06$ it is no longer significant.

Comparing the top and bottom row plots of Fig. 1 illustrates the strength of the method: For the two masses $a m=0.02$ and 0.04 , where the single correlators show a clear ghost contribution, the ghost appears in only one of the eigenvalues, while the other two are consistent with a dominant single exponential behavior. Our result shows that with the variational method ghost and proper states each dominate individual eigenvalues and the masses of the physical states can be fit without having to know or model the form of the ghost contribution.

Acknowledgements: The calculations were done on the Hitachi SR8000 at the Leibniz Rechenzentrum in Munich and we thank the LRZ staff for training and support. L. Y. G is supported by "Fonds zur Förderung der Wissenschaftlichen Forschung in Österreich", FWF, project P16823-N08. This work is supported by DFG and BMBF. We thank D. Aykroyd and H. Ramis for sharing their experience with removing ghosts.
[1] W. Bardeen, A. Duncan, E. Eichten, N. Isgur and H. Thacker, Phys. Rev. D 65 (2001) 014509.

[2] N. Mathur et al, Phys. Lett. B 605 (2005) 137.

[3] C. Michael, Nucl. Phys. B 259 (1985) 58.

[4] M. Lüscher and U. Wolff, Nucl. Phys. B 339 (1990) 222.

[5] S. Güsken, et al., Phys. Lett. B 227 (1989) 266; C. Best et al., Phys. Rev. D 56 (1997) 2743.

[6] T. Burch et al., Phys. Rev. D 70 (2004) 054502; Nucl. Phys. Proc. Suppl. 140 (2005) 284; Nucl. Phys. A 755 (2005) 481; Proc. Sci. LAT2005 (2005) 075; Proc. Sci. LAT2005 (2005) 097.

[7] S. Prelovsek, C. Dawson, T. Izubuchi, K. Orginos and A. Soni, Phys. Rev. D 70 (2004) 094503; T. Kunihiro, S. Muroya, A. Nakamura, C. Nonaka, M. Sekiguchi and H. Wada [SCALAR Collaboration], Phys. Rev. D 70 (2004) 034504; W. A. Bardeen, E. Eichten and
H. Thacker, Phys. Rev. D 69 (2004) 054502; C. McNeile and C. Michael [UKQCD Collaboration], Phys. Rev. D 63, 114503 (2001); W. J. Lee and D. Weingarten, Phys. Rev. D 61, 014015 (2000).

[8] C. Gattringer, Phys. Rev. D 63 (2001) 114501; C. Gattringer, I. Hip, and C.B. Lang, Nucl. Phys. B 597 (2001) 451.

[9] M. Lüscher and P. Weisz, Commun. Math. Phys. 97 (1985) 59; Err.: 98 (1985) 433; G. Curci, P. Menotti and G. Paffuti, Phys. Lett. B 130 (1983) 205, Err.: B 135 (1984) 516.

[10] C. Gattringer, R. Hoffmann, and S. Schaefer, Phys. Rev. D 65 (2002) 094503.

[11] C. Gattringer et al. [BGR Collaboration], Nucl. Phys. B 677 (2004) 3. 